\title{
SEM and TEM characterization of plastic deformation structures in Aluminum by EBSD, TKD, and PED-based orientation imaging techniques
}

\author{
P. Nowakowski ${ }^{1}$, J. Wiezorek ${ }^{2}$, V. Bathula ${ }^{2}$, S. Mielo ${ }^{1}$, S. Khanal ${ }^{2}$, C. Bonifacio ${ }^{1}$, and P. Fischione ${ }^{1}$ \\ ${ }^{1}$ E.A. Fischione Instruments, Inc., Export, PA, USA \\ ${ }^{2}$ Dept. of Mechanical Engineering and Materials Science, University of Pittsburgh, Pittsburgh, PA, USA
}

Diffraction-based analytical techniques for orientation imaging microscopy (OIM) with scanning and transmission electron microscope (SEM and TEM) instruments, such as electron backscatter diffraction (EBSD), transmission Kikuchi diffraction (TKD), and precession electron diffraction-assisted automated crystal orientation mapping (PED ACOM), offer powerful capabilities for spatially resolved studies of plastic deformation structures in materials [1]. These techniques are complementary regarding the respective combinations of the field-of-view and spatial resolution attainable. EBSD can gather data from very large areas (up to mm-scale, $\leq 10^{6} \mu^{2}$ ) with spatial resolution limited to $\sim 100 \mathrm{~nm}$ for Al, while TKD offers improved spatial resolution, $\sim 5$ to $10 \mathrm{~nm}$, within reduced maximum fields of view in the $\sim 10^{1}$ to $10^{2} \mu \mathrm{m}^{2}$ range. PED ACOM offers the highest spatial resolution, routinely $\sim 1$ to $3 \mathrm{~nm}$ in a field emission TEM, but is limited to analysis of localized areas in the $\sim 10^{0}$ to $10^{1} \mu \mathrm{m}^{2}$ range [2-4]. For deformation studies with these electron diffraction techniques, spatially resolved crystal orientation changes must be measured with high accuracy and precision [1]. Sample preparation can strongly affect the accuracy and precision attained in strain analyses [5]. Because EBSD signals originate from the top 30 to $50 \mathrm{~nm}$ of a sample, high-quality surface preparation is critical for accurate OIM-based strain analysis, while TKD and PED ACOM OIM require electron transparent specimens. For accurate study of deformation structures, sample preparation artifacts, e.g., contamination, lattice damage, and additional plastic deformation, have to be minimized or avoided. We have performed a comparative study of different sample preparation protocols on the deformation structures introduced to aluminum samples by controlled uniaxial compression at room temperature to obtain plastic strains of $0,4,6$, and 15\%. As a quantitative metric for deformation, the geometrically necessary dislocation (GND) density, $\rho_{\mathrm{GND}}$, has been derived from local orientation measurements under the assumption of negligible elastic stress [6]. We used two software analysis packages to determine $\rho_{\mathrm{GND}}$ from orientation maps: Atom [7], where $\rho_{\mathrm{GND}}$ is derived from the dislocation density tensor [6, 8]; and HKL CHANNEL5 [Oxford Instruments], where $\rho_{\mathrm{GND}}$ is calculated from representations of low angle boundaries [9, 10]. Four groups of sample preparation protocols have been applied for each deformed state of the $\mathrm{Al}$ and have been characterized by EBSD, TKD, and PED ACOM-based OIM:

- Samples for EBSD have been prepared by conventional mechanical polishing (MP) using colloidal silica (group 1), and subsequent additional $\mathrm{Ar}^{+}$broad ion beam (BIB) milling (group 2).

- Electron-transparent samples for TKD and PED ACOM have been obtained by $\mathrm{Ga}^{+}$focused ion beam (FIB) lift-out lamellae preparation and subsequent $\mathrm{Ar}^{+}$narrow ion beam milling (group 3), and BIB milling of MP conventional 3 mm diameter disk samples (group 4).

Figure 1 to 3, demonstrate effects from sample preparation in the $\rho_{\mathrm{GND}}$ measurements obtained by EBSD and TKD. The up to $\sim 25 \%$ higher $\rho_{\mathrm{GND}}$ in group 1 relative to group 2 samples is attributable to introduction of dislocations by abrasives during MP. Figure 2 shows the $\rho_{\mathrm{GND}}$ obtained from group 3 samples by TKD in the vicinity of a triple junction prior to compression. The TKD OIM of group 3 samples delivered a $\rho_{\mathrm{GND}}$ much larger than EBSD (groups 1 and 2). This could have resulted from a decrease in mapping step size [9] and/or 
orientation measurement determination uncertainties from distorted TKD pattern (off-axis detector used for TKD). Results of $\rho_{\mathrm{GND}}$ determination using PED ACOM will be discussed in relation to the TKD and EBSD measurements with a focus on sample preparation, diffraction pattern formation, and acquisition factors.

References:

[1] A Wilkinson, G Gonzalez, and DJ Dingley, Journal of Microscopy (U. K.) 169 (1993), p. 255.

[2] GC Sneddon, PW Trimby, and JM Cairney, Materials Science and Engineering R 110 (2016), p. 1.

[3] A Azdiar et al., Micron 103 (2017), p. 53.

[4] R Donatien et al., ACS Nano 7 (2013), p. 10887.

[5] SI Wright, MM Nowell, and DP Field, Microscopy and Microanalysis 17 (2011), p. 316.

[6] W Pantleon, Scripta Materialia 58 (2008), p. 994.

[7] B Beausir, JJ Fundenberger, ATOM - Analysis Tools for Orientation Maps.

[8] B Beausir and C Fressengeas, International Journal of Solids and Structures 50 (2013), p. 137.

[9] C Moussa et al., Ultramicroscopy 179 (2017), p. 63.

[10] WT Read and W Shockley, Physical Review 78 (1950), p. 275.

[11] This work received support from the National Science Foundation, NSF-1607922.

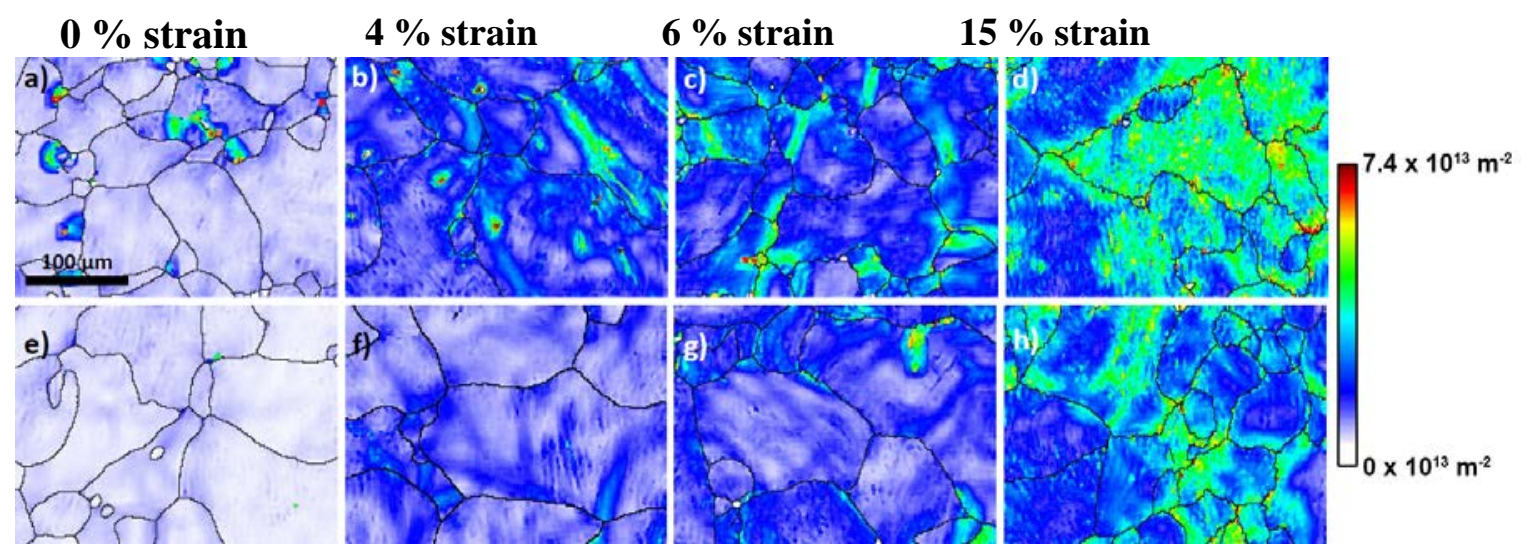

Figure 1. $\rho_{\mathrm{GND}}$ map determined from EBSD data using HKL Channel5 software (step size $1.5 \mu \mathrm{m}$ ). Group 1 samples (a-d) prepared by mechanical polishing using silica colloidal; group 2 samples (e-h) prepared by $\mathrm{Ar}^{+}$broad ion beam at $4 \mathrm{keV}$ and cleaned at $1 \mathrm{keV}$.

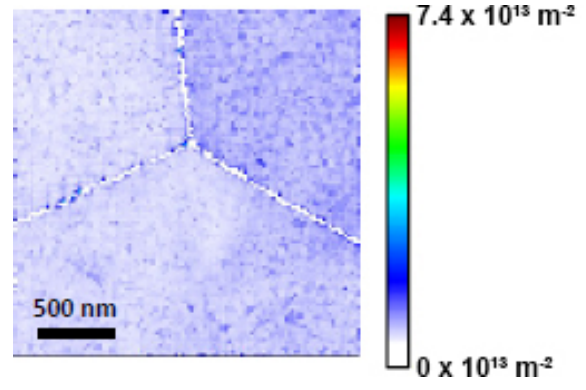

Figure 2. High-resolution TKD maps acquired with step size $20 \mathrm{~nm}$ from $\mathrm{Al}$ with $0 \%$ strain (group 3). Lamella prepared by $\mathrm{Ga}^{+}$ FIB (30 keV followed by $5 \mathrm{keV}$ ) and cleaned by $\mathrm{Ar}^{+}$narrow ion beam milling at $500 \mathrm{eV}$.

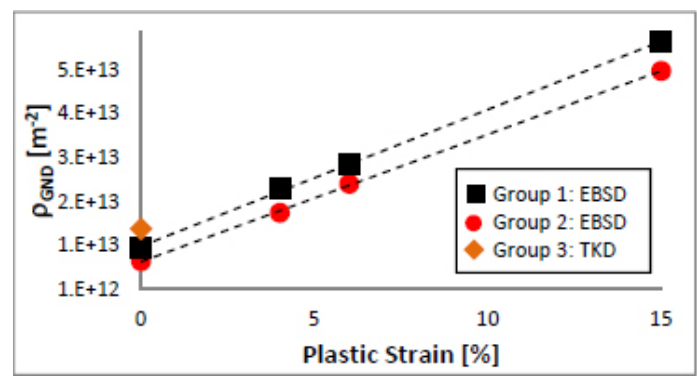

Figure 3. $\rho_{\mathrm{GND}}$ calculated from EBSD and TKD data. 